\title{
Facile Access to Polysubstituted Indoles via Three Component One - Pot Reactions
}

\author{
ABOLFAZL ESHGHI MOVAHHED* and MOHAMMAD HOSSEIN MOSSLEMIN
}

Department of Chemistry, Islamic Azad University, Yazd Branch, P.O. Box 89195-155, Yazd, Iran

abmovahed@yahoo.com

Received 30 May 2012 / Accepted 22 June 2012

\begin{abstract}
A simple and efficient method for the synthesis of a novel series of 2, 3, 5 trisubstituted indoles in high yields is discribed from an one-pot, three-component reaction of $p$-aniline, the phenylglyoxals and barbituric acid or dimedon in HOAc.
\end{abstract}

Keywords: Trisubstituted indoles synthesis, One-pot and three-component reaction, Aniline, phenylglyoxals, Barbituric acid, dimedon

\section{Introduction}

Multi-component reactions (MCRs) play an important role in organic chemistry. The significant contribution of MCRs to the state of the art of modern organic chemistry and their potential use in complex organic syntheses ${ }^{1}$. Indole and its derivatives are known as important intermediates in organic synthesis and pharmaceutical chemistry ${ }^{2}$. These compounds exhibit various physiological properties and pharmacological activities ${ }^{2}$ such as: beneficial estrogen metabolism promoter ${ }^{3}$, inhibitor for human prostate cancer cells and radical scavengers ${ }^{4}$. Therefore, development of facile and practical approaches to the synthesis of these classes of compounds has been investigated by a number of organic and pharmaceutical chemists. Although these are efficient process and have been used widely for the preparation of substituted indoles ${ }^{5-8}$, some of these methods involve two or more sequential synthetic steps, the use of harsh reaction conditions that give low yields and drastic condition for catalyst preparation. These limitations of the current available synthetic approaches to substituted indole derivatives prompted us to develop an alternative synthesis of indole derivatives. Here, we report a new, simple and general synthetic method of substituted indoles.

\section{Experimental}

All chemicals were purchased from Merck, Fluka and Aldrich and used without further purification. The products were characterized by their melting point and spectral data. All yields refer to isolated products. ${ }^{1} \mathrm{H}$ NMR and ${ }^{13} \mathrm{C}-\mathrm{NMR}$ spectra were recorded on a Bruker 
DRX-400 and 500 in DMSO-d6 relative to TMS as an internal standard. IR spectra were run on a shimadzu IR- 470 spectrometer. Elemental analyses were performed of using a Heraeus CHN-O-Rapid analyzer.

General procedure for the preparation of compounds $(\mathbf{4 a}-\mathbf{j})$

A mixture of aniline $(1 \mathrm{mmol})$, phenylglyoxal $(1 \mathrm{mmol})$ and dimedone or barbituric acid ( $1 \mathrm{mmol})$ in HOAc $(10 \mathrm{~mL})$ was refluxed for $4 \mathrm{~h}$. After cooling, the precipitated solid was isolated by filtration and it was purified by recrystallisation in ethanol.

6-Hydroxy-5-(5-nitro-3-(4-nitrophenyl)-1H-indol-2-yl)pyrimidine-2,4(1H,3H)dione (4a)

Brown solid; mp $365{ }^{\circ} \mathrm{C}$ dec. ${ }^{1} \mathrm{H}$ NMR (DMSO) (400 MHz): $\delta=7.6(1 \mathrm{H}, \mathrm{d}, J=8.8), 7.91$ $(2 \mathrm{H}, \mathrm{d}, J=8.8), 8.07(1 \mathrm{H}, \mathrm{dd}, J=8.8,2), 8.23(1 \mathrm{H}, \mathrm{d}, J=2), 8.34(2 \mathrm{H}, \mathrm{d}, J=8.8), 10.95$ $(2 \mathrm{H}, \mathrm{s}), 12.44(1 \mathrm{H}, \mathrm{s}) .{ }^{13} \mathrm{C}$ NMR (DMSO): $\delta=83.21,108.4,112.48,117.21,118.33,124.5$, $128.11,129.64,138.13,138.85,140.33,141.39,146.91,150.84,162.3$. IR (KBr) $v=3750$, 3125, 1726, 1627, 1511, $1338 \mathrm{~cm}^{-1}$. Anal. Calcd (\%) for $\mathrm{C}_{18} \mathrm{H}_{11} \mathrm{~N}_{5} \mathrm{O}_{7}$ : (409.31): C, 52.82; $\mathrm{H}$, 2.71; N, 17.11; Found: C, 52.94; H, 2.75; N, 17.02. MS: $m / z(\%)=409(42)[\mathrm{M}+]$.

6-Hydroxy-5-(5-methyl-3-(4-nitrophenyl)-1H-indol-2-yl)pyrimidine-2,4(1H,3H)dione (4b)

Light green solid; mp $360{ }^{\circ} \mathrm{C}$ dec. ${ }^{1} \mathrm{H}$ NMR (DMSO) (400 MHz): $\delta=2.35(3 \mathrm{H}, \mathrm{s}), 7(1 \mathrm{H}, \mathrm{dd}$, $J=7.2,1), 7.04(1 \mathrm{H}, \mathrm{d}, J=1), 7.22(1 \mathrm{H}, \mathrm{d}, J=7.2), 7.86(2 \mathrm{H}, \mathrm{d}, J=8.8), 8.27(2 \mathrm{H}, \mathrm{d}, J=8.8$, ), 10.91(2H, s), $12.18(1 \mathrm{H}, \mathrm{s}) .{ }^{13} \mathrm{C}$ NMR (DMSO): $\delta=21.67,84.48,105.76,111.67,119.72$, $124.33,124.97,127.3,128.35,130.57,134.51,135.76,140.33,145.95,150.97,162.45$. IR $(\mathrm{KBr}) \mathrm{v}=3745,3420,1721,1628,1598,1337 \mathrm{~cm}^{-1}$. Anal. Calcd $(\%)$ for $\mathrm{C}_{19} \mathrm{H}_{14} \mathrm{~N}_{4} \mathrm{O}_{5}$ : (378.34): C, 60.32; H, 3.73; N, 14.81; Found: C, 60.36; H, 3.75; N, 14.92. MS: $m / z(\%)=378(61)[\mathrm{M}+]$.

5-(3-(4-Chlorophenyl)-5-methyl-1H-indol-2-yl)-6-hydoxypyrimidine-2,4(1H,3H)dione $(4 \mathrm{c})$

Cream solid; mp $362{ }^{\circ} \mathrm{C} \mathrm{dec} .{ }^{1} \mathrm{H}$ NMR (DMSO) (400 MHz): $\delta=2.33(3 \mathrm{H}, \mathrm{s}), 6.92(1 \mathrm{H}, \mathrm{dd}$, $J=8,1), 6.98(1 \mathrm{H}, \mathrm{d}, J=1), 7.27(1 \mathrm{H}, \mathrm{d}, J=8), 7.45(2 \mathrm{H}, \mathrm{d}, J=8.4), 7.6(2 \mathrm{H}, \mathrm{d}, J=8.4), 10.72$ $(2 \mathrm{H}, \mathrm{s}), 11.3(1 \mathrm{H}, \mathrm{s}) .{ }^{13} \mathrm{C}$ NMR (DMSO): $\delta=21.41,83.82,105.8,116.31,117.4,124.82$, $127.98,128.75,129.22,130.45,133.22,134.35,135.7,139.3,150.8,162.38$. IR (KBr) $v=3735,3345,1686,1578,1387,1080 \mathrm{~cm}^{-1}$. Anal. Calcd (\%) for $\mathrm{C}_{19} \mathrm{H}_{14} \mathrm{ClN}_{3} \mathrm{O}_{3}:(367.79): \mathrm{C}$, 62.05; H, 3.84; N, 11.43; Found: C, 61.92; H, 3.75; N, 11.55. MS: $m / z(\%)=367(52)[\mathrm{M}+]$.

5-(3-(4-Chlorophenyl)-5-nitro-1H-indol-2-yl)-6-hydroxypyrimidine-2,4(1H,3H)-

dione (4d)

Brown solid; mp $360{ }^{\circ} \mathrm{C}$ dec. ${ }^{1} \mathrm{H}$ NMR (DMSO) (400 MHz): $\delta=7.55(1 \mathrm{H}), 7.55(2 \mathrm{H}, \mathrm{d}, J=8)$, $7.65(2 \mathrm{H}, \mathrm{d}, J=8), 8.03(1 \mathrm{H}, \mathrm{dd}, J=8,1.2), 8.16(1 \mathrm{H}, \mathrm{d}, J=1.2), 10.93(2 \mathrm{H}, \mathrm{s}), 12.24(1 \mathrm{H}, \mathrm{s})$. ${ }^{13} \mathrm{C}$ NMR (DMSO): $\delta=83.38,105.72,112.08,116.53,117.59,129.04,129.25,129.9$, $131.27,133.29,139.54,140.02,141.21,150.81,162.41$. IR $(\mathrm{KBr}) v=3738,3225,1695$, 1609, 1484, $1337 \mathrm{~cm}^{-1}$. Anal. Calcd (\%) for $\mathrm{C}_{18} \mathrm{H}_{11} \mathrm{ClN}_{4} \mathrm{O}_{5}$ : (398.76): C, 54.22; H, 2.78; N, 14.05; Found: C, 54.11; H, 2.82; N, 13.93. MS: $m / z(\%)=398(37)[\mathrm{M}+]$.

5-(5-Chloro-3-phenyl)-1H-indol-2-yl)-6-hydroxypyrimidine-2,4(1H,3H)-dione (4e)

Brown solid; mp $365{ }^{\circ} \mathrm{C} \mathrm{dec} .{ }^{1} \mathrm{H}$ NMR (DMSO) $(400 \mathrm{MHz}): \delta=6.92(1 \mathrm{H}, \mathrm{t}, J=8), 7.16(1 \mathrm{H}$, $\mathrm{dd}, J=8.8,3), 7.28(1 \mathrm{H}, \mathrm{d}, J=3), 7.45(1 \mathrm{H}, \mathrm{d}, J=8.4), 7.86(2 \mathrm{H}, \mathrm{t}, J=8), 8.31(2 \mathrm{H}, \mathrm{d}, J=8.8)$, 
10.94(2H, s), 11.94(1H, s). ${ }^{13} \mathrm{C}$ NMR (DMSO): $\delta=83.98,105.51,113.51,119.34,121.97$, $123.63,124.43,127.74,128.79,129.76,131.43,136.22,139.59,150.86,162.45 . \mathrm{IR}(\mathrm{KBr})$ $v=3721,3223,1676,1595,1450,1345 \mathrm{~cm}^{-1}$. Anal. Calcd (\%) for $\mathrm{C}_{18} \mathrm{H}_{12} \mathrm{ClN}_{3} \mathrm{O}_{3}:(353.8): \mathrm{C}$, $61.11 ; \mathrm{H}, 3.42 ; \mathrm{N}, 11.88$; Found: C, 61.23; H, 3.47; N, 11.74. MS: $m / z(\%)=353(48)[\mathrm{M}+]$.

6-Hydroxy-5-(5-nitro-3-phenyl-1H-indol-2-yl)pyrimidine-2,4(1H,3H)-dione (4f)

Light green solid; mp $360{ }^{\circ} \mathrm{C} \mathrm{dec} .{ }^{1} \mathrm{H}$ NMR (DMSO) (400 MHz): $\delta=7.36(1 \mathrm{H}, \mathrm{t}, J=7.6), 7.47$ $(2 \mathrm{H}, \mathrm{t}, J=7.6), 7.55(1 \mathrm{H}, \mathrm{d}, J=8.8), 7.65(2 \mathrm{H}, \mathrm{d}, J=7.6), 8.02(1 \mathrm{H}, \mathrm{dd}, J=8.8,2), 8.15(1 \mathrm{H}, \mathrm{d}$, $\mathrm{J}=2), 10.91(2 \mathrm{H}, \mathrm{s}), 12.18(1 \mathrm{H}, \mathrm{s}) .{ }^{13} \mathrm{C}$ NMR (DMSO): $\delta=83.56,105.13,112,116.35,117.39$, $127.39,128.64,129.17,130.12,132.38,140.02,140.84,141.13,150.85,162.31 . \mathrm{IR}(\mathrm{KBr})$ $v=3730,3405,1696,1607,1469,1333 \mathrm{~cm}^{-1}$. Anal. Calcd $(\%)$ for $\mathrm{C}_{18} \mathrm{H}_{12} \mathrm{~N}_{4} \mathrm{O}_{5}$ : (364.31): $\mathrm{C}$, 59.34; H, 3.32; N, 15.38; Found: C, 59.42; H, 3.38; N, 15.25. MS: $m / z(\%)=364(70)[\mathrm{M}+]$.

2-(5-Chloro-3-(4-chlorophenyl)-1H-indol-2-yl)-5,5-dimethylcyclohexa-1,3-diene1,3-diol (4g)

Cream solid; mp $290{ }^{\circ} \mathrm{C}$ dec. ${ }^{1} \mathrm{H}$ NMR (DMSO) $(500 \mathrm{MHz}): \delta=1.07(3 \mathrm{H}, \mathrm{s}), 1.12(3 \mathrm{H}, \mathrm{s})$, 2.2-2.43( 4H, br), 7.24(1H, d, $J=1.73), 7.04(1 \mathrm{H}, \mathrm{dd}, J=8.5,2), 7.34(1 \mathrm{H}, \mathrm{d}, J=8.45), 7.42$ $(2 \mathrm{H}, \mathrm{d}, J=8.56), 7.54(2 \mathrm{H}, \mathrm{d}, J=8.57), 10.25(1 \mathrm{H}, \mathrm{s}, \mathrm{OH})\left(\right.$ exchange with $\left.\mathrm{D}_{2} \mathrm{O}\right), 11.53(1 \mathrm{H}$, $\mathrm{s}, \mathrm{NH}) .{ }^{13} \mathrm{C}$ NMR $\left(\mathrm{CDCl}_{3}\right): \delta=28.93,29.13,32.47,106.34,108.58,113.47,119.27,122.14$, $124.13,129.21,129.3,131.48,132.76,132.8,135.55,136.58 . \mathrm{IR}(\mathrm{KBr}) v=3380,3110$, $1608,1465,1317,1019 \mathrm{~cm}^{-1}$. Anal. Calcd (\%) for $\mathrm{C}_{22} \mathrm{H}_{19} \mathrm{Cl}_{2} \mathrm{NO}_{2}$ : (400.3): C, 66.01; H, 4.78; N, 3.5; Found: C, 66.12; H, 4.83; N, 3.41. MS: $m / z(\%)=399(100)[\mathrm{M}+]$.

2-(3-(4-Chlorophenyl)-5-nitro-1H-indol-2-yl)-5,5-dimethylcyclohexa-1,3-diene-1,3$\operatorname{diol}(4 h)$

Green solid; mp $300{ }^{\circ} \mathrm{C}$ dec. ${ }^{1} \mathrm{H}$ NMR (DMSO) (500 MHz): $\delta=1.08(3 \mathrm{H}, \mathrm{s}), 1.14(3 \mathrm{H}, \mathrm{s})$, 2.41(2H, br), 2.57(2H, br), 7.47(2H, d, $J=8.53), 7.50(1 \mathrm{H}, \mathrm{d}, J=8.83), 7.57(2 \mathrm{H}, \mathrm{d}, J=8.55)$, $7.97(1 \mathrm{H}, \mathrm{dd}, J=8.75,2.2), 8(1 \mathrm{H}, \mathrm{d}, J=2), 10.5(1 \mathrm{H}, \mathrm{s}, \mathrm{OH})\left(\right.$ exchange with $\left.\mathrm{D}_{2} \mathrm{O}\right), 12.12(1 \mathrm{H}$, $\mathrm{s}, \mathrm{NH}) .{ }^{13} \mathrm{C} \mathrm{NMR}\left(\mathrm{CDCl}_{3}\right): \delta=28.84,29.14,32.54,107.83,109.04,112.44,116.96,117.78$, $129.45,129.47,129.64,132.08,133.42,138.56,140.38,141.36$. IR $(\mathrm{KBr}) v=3460,3055$, $1613,1472,1318,1027 \mathrm{~cm}^{-1}$. Anal. Calcd (\%) for $\mathrm{C}_{22} \mathrm{H}_{19} \mathrm{ClN}_{2} \mathrm{O}_{4}$ : (410.9): C, 64.31; H, 4.66; N, 6.82; Found: C, 64.19; H, 4.73; N, 6.88. MS: $m / z(\%)=410(90)[\mathrm{M}+]$.

2-(5-Chloro-3-(4-nitrophenyl)-1H-indol-2-yl)-5,5-dimethylcyclohexa-1,3-diene-1,3diol (4i)

Yellow solid; mp $350{ }^{\circ} \mathrm{C}$ dec. ${ }^{1} \mathrm{H}$ NMR (DMSO) (500 MHz): $\delta=1.11(3 \mathrm{H}, \mathrm{s}), 1.13(3 \mathrm{H}, \mathrm{s})$, 2.32(2H, br), 2.55(2H, br), 7.08(1H, d, $J=2), 7.12(1 \mathrm{H}, \mathrm{dd}, J=8.5,2), 7.39(1 \mathrm{H}, \mathrm{d}, J=8.5)$, $7.72(2 \mathrm{H}, \mathrm{d}, J=8.5), 8.22(2 \mathrm{H}, \mathrm{d}, J=8.5), 10.27(1 \mathrm{H}, \mathrm{s}, \mathrm{OH})\left(\right.$ exchange with $\left.\mathrm{D}_{2} \mathrm{O}\right), 12(1 \mathrm{H}, \mathrm{s}$, $\mathrm{NH}) .{ }^{13} \mathrm{C}$ NMR $\left(\mathrm{CDCl}_{3}\right): \delta=28.87,29.26,32.49,108.38,109.14,113.87,119.82,123.31$, $124.52,124.65,127.99,131.25,135.24,136.1,140.41,146.71$. IR $(\mathrm{KBr}) v=3360,3075$, 1599, 1507, 1325, $1022 \mathrm{~cm}^{-1}$. Anal. Calcd (\%) for $\mathrm{C}_{22} \mathrm{H}_{19} \mathrm{ClN}_{2} \mathrm{O}_{4}$ : (410.9): C, 64.31; H, 4.66; $\mathrm{N}, 6.82$; Found: C, 64.42; H, 4.71; N, 6.91. MS: $m / z(\%)=410(92)[\mathrm{M}+]$.

\section{5,5-Dimethyl-2-(5-methyl-3-(4-nitrophenyl)-1H-indol-2-yl)-cyclohexa-1,3-diene- 1,3 -diol $(\mathbf{4 j})$}

Orange solid; mp $350{ }^{\circ} \mathrm{C}$ dec. ${ }^{1} \mathrm{H}$ NMR (DMSO) (500 MHz): $\delta=1.11(\mathrm{H}, \mathrm{s}), 1.15(3 \mathrm{H}, \mathrm{s}), 2.30$ $(5 \mathrm{H}, \mathrm{s}), 2.53(2 \mathrm{H}, \mathrm{s}), 6.88(1 \mathrm{H}, \mathrm{s}), 6.94(1 \mathrm{H}, \mathrm{dd}, J=8.3,0.8), 7.26(1 \mathrm{H}, \mathrm{d}, J=8.2), 7.75(2 \mathrm{H}, \mathrm{d}, J=8.87)$, 
8.19(2H, d, $J=8.77), 10.25(1 \mathrm{H}, \mathrm{s}, \mathrm{OH})\left(\right.$ exchange with $\left.\mathrm{D}_{2} \mathrm{O}\right), 11.43(1 \mathrm{H}, \mathrm{s}, \mathrm{NH}) .{ }^{13} \mathrm{C}$ NMR $\left(\mathrm{CDCl}_{3}\right): \delta=22.16,28.75,29.45,32.5,46.7,52.4,109.16,109.27,111.98,120.29,124.61$, $125.24,127.54,128.33,130.47,133.53,136.2,141.08,146.19,172.54,197.47:$ IR $(\mathrm{KBr}) \mathrm{v}=$ $3380,3120,1611,1503,1337,1022 \mathrm{~cm}^{-1}$. Anal. Calcd (\%) for $\mathrm{C}_{23} \mathrm{H}_{22} \mathrm{~N}_{2} \mathrm{O}_{4}:(390.4): \mathrm{C}, 70.75 ; \mathrm{H}$, 5.68; N, 7.17; Found: C, 70.62; H, 5.71; N, 7.27. MS: $m / z(\%)=390(81)[\mathrm{M}+]$.

\section{Results and Discussion}

Our studies were initiated by heating a solution of phenylglyoxals $\mathbf{3 a}-\mathbf{c}$, anilines $\mathbf{2 a - c}$ and barbituric acid in the presence of glacial acetic acid under reflux for 4 hour (Scheme 1).

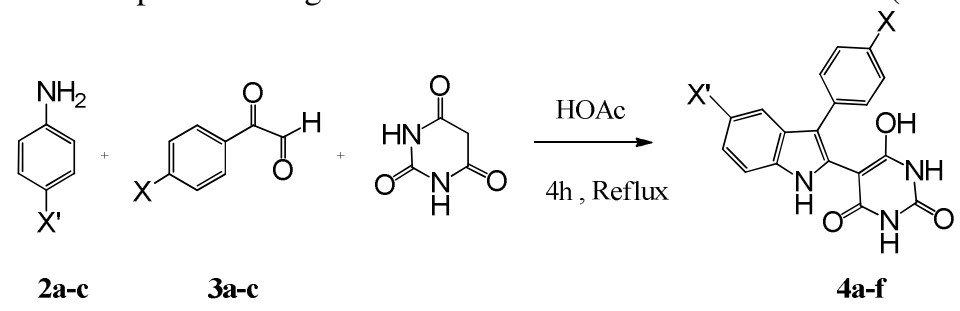

Scheme 1. Indole synthesis of aniline, phenylglyoxal and barbituric acid

Then the trisubstituted indoles $\mathbf{4 g}-\mathbf{j}$ were synthesized by refluxing of phenylglyoxals 3a-c, anilines 2a-c and dimedon in same condition (Scheme 2).<smiles>[X]c1ccc(N)cc1</smiles>

2a-c<smiles>[X]c1ccc(C=O)cc1</smiles>

3a-c<smiles>[X]c1ccc(-c2c(C3=C(O)CC(C)(C)CC3=O)[nH]c3ccc([X])cc23)cc1</smiles>

$4 \mathrm{~g}-\mathbf{j}$

Scheme 2. Indole synthesis of aniline, phenylglyoxal and dimedon

After completion and simple filtration, products $\mathbf{4 a - j}$ were obtained in $>83 \%$ yields. The results are presented in Table 1. Phenylglyoxals were synthesized by oxidation of acetophenones with selenium dioxide in the presence of dioxin or ethyl alcohol as solvent ${ }^{9}$.

Table 1. Trisubstituted Indoles prepared from anilines 2a-c, phenylglyoxals 3a-c and barbituric acid or dimedon.

\begin{tabular}{ccccc}
\hline Entry & $\mathrm{X}$ (glyoxal) & $\mathrm{X}^{\prime}$ (aniline) & $\mathbf{4}$ & Yield \% \\
\hline 1 & $\mathrm{NO}_{2}$ & $\mathrm{NO}_{2}$ & $\mathbf{4 a}$ & 86 \\
2 & $\mathrm{NO}_{2}$ & $\mathrm{CH}_{3}$ & $\mathbf{4 b}$ & 91 \\
3 & $\mathrm{Cl}$ & $\mathrm{CH}_{3}$ & $\mathbf{4 c}$ & 89 \\
4 & $\mathrm{Cl}$ & $\mathrm{NO}_{2}$ & $\mathbf{4 d}$ & 85 \\
5 & $\mathrm{H}$ & $\mathrm{Cl}$ & $\mathbf{4 e}$ & 85 \\
6 & $\mathrm{H}$ & $\mathrm{NO}_{2}$ & $\mathbf{4 f}$ & 83 \\
7 & $\mathrm{Cl}$ & $\mathrm{Cl}$ & $\mathbf{4 g}$ & 86 \\
8 & $\mathrm{Cl}$ & $\mathrm{NO}_{2}$ & $\mathbf{4 h}$ & 85 \\
9 & $\mathrm{NO} 2$ & $\mathrm{Cl}^{2}$ & $\mathbf{4 i}$ & 89 \\
10 & $\mathrm{NO} 2$ & $\mathrm{CH}_{3}$ & $\mathbf{4 j}$ & 92 \\
\hline
\end{tabular}

The structure of compounds $\mathbf{4} \mathbf{a}-\mathbf{j}$ was proved on the basis of the mass spectra, elemental analysis and ${ }^{1} \mathrm{H}$ and ${ }^{13} \mathrm{C}$ NMR spectrum. The mass spectra of these compounds displayed 
molecular ion peaks at appropriate $\mathrm{m} / \mathrm{z}$ values. The elemental analysis of these compounds proved the structure of compounds. Since synthesized structures have tautomeric forms, some of ${ }^{1} \mathrm{H}$ NMR and ${ }^{13} \mathrm{C}$ NMR spectrums are broad and are not seen (Scheme 3 ).<smiles>[X]c1ccc2[nH]c(C3=C(O)CCCC3=O)c([R10])c2c1</smiles><smiles>[X]c1ccc2[nH]c(C3=C(O)CCCC3=O)c([R12])c2c1</smiles>

Scheme 3. Tautomeric forms of $4 \mathbf{a}-\mathbf{j}$

Furthermore we think that rotation of dimedone or barbituric acid ring is effective (Scheme 4). For instance the ${ }^{1} \mathrm{H}$ NMR spectrum $\mathbf{4 b}$ exhibited a broad resonance at 10.91 for two protons of $\mathrm{NH}$ barbituric acid and this did not exhibit a resonance for $\mathrm{OH}$.<smiles>[Y]c1ccc2[nH]c(C=CC3=C(O)CCCC3=O)c(P)c2c1</smiles>

Scheme 4. Rotation of barbituric acid/ dimedon ring of $\mathbf{4 a - j}$

The ${ }^{13} \mathrm{C}$ NMR spectrum $4 \mathrm{~b}$ exhibited a broad and weak resonance at $162.45 \mathrm{ppm}$ for carbonyl group. Also the ${ }^{1} \mathrm{H}$ NMR spectrum $4 \mathrm{~h}$ exhibited a broad resonance at 10.5 for $\mathrm{OH}$ (disappeared after addition of a few drops of $\mathrm{D}_{2} \mathrm{O}$ ) and two broad and weak resonances at 2.41 and 2.57 for $\mathrm{CH}_{2}$ dimedon. The ${ }^{13} \mathrm{C}$ NMR spectrum 4 h did not exhibit resonances for $\mathrm{CH}_{2}, \mathrm{COH}$ and $\mathrm{CO}$ dimedon. ${ }^{1} \mathrm{H}$ and ${ }^{13} \mathrm{C}$ NMR spectrums repeated at 20, 50, 70, 90 and $100{ }^{\circ} \mathrm{C}$ but there was not appear the resonance for not seen resonances. The tautomerism of barbituric acid and substituted barbituric acids has been extensively investigated ${ }^{10}$.

In conclusion, we have successfully developed a novel, direct and efficient and environmentally friendly one-pot three-component coupling reaction to syntheses $2,3,5$ trisubstituted indoles in high yields by employing the anilines, the phenylglyoxals and barbituric acid/ dimedon. The simplicity of this experiment and work up are noteworthy.

\section{Acknowledgement}

We gratefully acknowledge financial support from the Research Council of Islamic Azad University, Yazd branch.

\section{References}

1. Zhu J and Bienayme H, Multicomponent Reactions, Wiley-Vch Verlag GmbH \& Co, 2005.

2. Sundberg R J, The Chemistry of Indoles, Academic Press, New York, 1970, 1-3.

3. Chang Y C, Riby J, Chang G, Peng B, Firestone G L and Bjeldanes L F, Biochem Pharmacol., 1999, 58, 825-834.

4. Benabadji S H, Wen R, Zheng J, Dong X and Yuan S, Acta Pharmacol Sin., 2004, 25, 666.

5. Humphrey G R and Kuethe J T, Chem Rev., 2006, 106, 2875-2911.

6. (a) Stuard D R, Villemure E and Fagnou K, J Am Chem Soc., 2007, 129, 12072-12073; (b) Zhao J, Zhang Y and Cheng K, J Org Chem., 2008, 73, 7428; (c) Jia Y and Zhu J, J Org Chem., 2006, 71, 7826; (d) Bellina F, Benelli F and Rossi R, J Org Chem., 2008, 73, 5529; (e) Kaspar L K and Ackermann L, Tetrahedron, 2005, 61, 11311-11316; (f) Queiroz M J R P, Abreu A S, Castanheira E M S and Ferreira P M T, Tetrahedron, 2007, 63, 2215. 
7. (a) Hiroya K, Matsumoto S and Sakamoto T, Org Lett., 2004, 6, 2953-2956; (b) Lu B Z, Zhao W, Wei H-X, Dufour M, Farina V and Senanayake C H, Org Lett., 2006, 8, 3271-3274.

8. (a) Yue D, Yao T and Larock R C, J Org Chem., 2006, 71, 62-69; (b) Larock R C, Yum E K and Refvik M D, J Org Chem., 1998, 63, 7652-7662; (c) Yue D and Larock R C, Org Lett., 2004, 6, 1037; (d) Zhou H, Liao X and Cook J M, Org Lett., 2004, 6, 249-252; (e) Ohno H, Ohta Y, Oishi S and Fujii N, Angew Chem Int Ed., 2007, 46(13), 2295-2298.

9. Furniss B S, Hannaford A J, Smith P W G and Tatchell A R, Vogel's Textbook of Practical Organic Chemistry, $5^{\text {th }}$ Edn., Wiley, New York, 1989, 629.

10. (a) Ralhan S and Ray N K, Theochem., 2003, 634(1), 83-88; (b) Oguz Funda S and Dogan L, Spectroscopy Letters., 2004, 37(6), 607-618; (c) Krasnov K A, Kartsev V G and Santarovich E E, Chem Heterocycl Compounds., 2002, 38, 702-709; (d) AnaryAbbasinejad M, Kamali-Gharamaleki M and Hassanabadi A, J Chem Res., 2007, 10, 594-597. 